\title{
A DANIELL-STONE APPROACH TO THE GENERAL DENJOY INTEGRAL
}

\author{
CORNEL LEINENKUGEL
}

(Communicated by R. Daniel Mauldin)

\begin{abstract}
In this note we shall introduce a, as far as we know, new kind of derivative (diagonal derivative), characterizing a certain class of functions $\mathscr{E}_{d}$ and a generalized Daniell integral $I_{d}$ on this class. We follow Leinert and König to obtain a class of integrable functions $\mathscr{L}_{d}^{1}$ belonging to $\mathscr{E}_{d}$, using the method of Daniell-Stone integration without the lattice condition as described in [1] or similarly in [3]. Our main purpose is to show that we obtain exactly the Denjoy integrable functions.
\end{abstract}

We proceed in three steps. In $\S 1$, with a little change in a minor detail, we briefly put together the most important things about Leinert's method of Daniell-Stone integration without the lattice condition as described in [1]. In $\S 2$, after some other prerequisites, we introduce the notion of diagonal differentiability, leading to a class of integrable functions $\mathscr{L}_{d}^{1}$ by using $\S 1$. A more detailed comparison of these functions with the Lebesgue integrable functions follows. In $\S 3$, we give a new characterization of ACG-functions in which diagonal differentiability is involved. As a consequence, the functions of $\mathscr{L}_{d}^{1}$ turn out to be exactly the Denjoy integrable functions.

Note that in the theory of Perron and Denjoy a generalized form of differentiability describes the integrable functions in a direct way (as generalized derivatives), whereas in our theory a notion of differentiability is used to define the function space $\mathscr{E}_{d}$ from which the integrable functions are obtained by a Daniell-Stone procedure.

\section{Daniell-Stone integration Without the lattice Condition}

Let us first describe Leinert's method of avoiding the lattice condition in Daniell-Stone integration.

Given a nonvoid set $X$ and a real vector space $\mathscr{E}$ (not necessarily a lattice) of functions $X \rightarrow \mathbf{R}$, a functional $I: \mathscr{E} \rightarrow \mathbf{R}$ is called a Daniell integral, if it is linear, positive (i.e. $I(f) \geq 0$ whenever $f \geq 0$ ) and if it satisfies the following condition (continuity from below): For arbitrary $f \in \mathscr{E}, f_{n} \in \mathscr{E}^{+}$we have

$$
\sum_{1}^{\infty} f_{n} \geq f \Rightarrow \sum_{1}^{\infty} I\left(f_{n}\right) \geq I(f) .
$$

Received by the editors August 3, 1989.

1980 Mathematics Subject Classification (1985 Revision). Primary 26A39; Secondary 26A24. 
As usual in integration theory, the value $+\infty$ is not excluded when dealing with sums of nonnegative numbers or functions. Here and further on, for a set $\mathscr{F}$ of functions $X \rightarrow \overline{\mathbf{R}}$, we define $\mathscr{F}+$ to be the set of all $f \in \mathscr{F}$ with $f \geq 0$. On the set $\mathscr{P}$ of all functions $X \rightarrow[0, \infty]$, the functional $\bar{I}$ is defined by

$$
\bar{I}(f):=\inf \left\{\sum_{1}^{\infty} I\left(f_{n}\right) \mid f_{n} \in \mathscr{E}^{+}, \sum_{1}^{\infty} f_{n} \geq f\right\} \in[0, \infty] .
$$

Note that $\inf \varnothing=\infty$. For an arbitrary $f: X \rightarrow \overline{\mathbf{R}}$ the $(I-)$ Norm of $f$ is $\|f\|:=\bar{I}(|f|)$. The functional $\bar{I}$ is countably subadditive, i.e.

$$
\bar{I}\left(\sum_{1}^{\infty} f_{n}\right) \leq \sum_{1}^{\infty} \bar{I}\left(f_{n}\right) \quad \forall f_{1}, f_{2}, \cdots \in \mathscr{P} .
$$

A function $f: X \rightarrow \overline{\mathbf{R}}$ is called $(I-)$ integrable, if for every $\varepsilon>0$ there is $g \in \mathscr{E}$ with $\|f-g\|<\varepsilon$. The space of all integrable functions is denoted by $\mathscr{L}^{1}=\mathscr{L}^{1}(X, \mathscr{E}, I)$. This differs a little from the definition in [1], since there the approximating functions $g \in \mathscr{E}$ are required to have finite norm. But this is not at all essential, so the following remains true by the same argument as in [1]. If $f \in \mathscr{L}^{1}$ and $g_{n} \in \mathscr{E}$ are functions with $\left\|f-g_{n}\right\| \rightarrow 0$, then the $(I-)$ integral of $f$ over $X$ is well defined by

$$
\int f d I:=\lim _{n \rightarrow \infty} I\left(g_{n}\right) \in \mathbf{R} .
$$

A function $g: X \rightarrow \overline{\mathbf{R}}$ is called a null function if $\|g\|=0$. A set $A \subset X$ is called a null set if its characteristic function $\chi_{A}$ is a null function. A property $Q$ is said to hold almost everywhere (a.e.) if it holds outside some null set.

Let us recall the following proposition from [1].

1. Proposition. For $f, g: X \rightarrow \overline{\mathbf{R}}$ we have:

(i) $f=g$ a.e. $\Rightarrow\|f\|=\|g\|$.

(ii) $f \in \mathscr{L}^{1}$ and $f=g$ a.e. $\Rightarrow g \in \mathscr{L}^{1}$ and $\int f d I=\int g d I$.

(iii) $f \in \mathscr{L}^{1} \Rightarrow\{x \in X|| f(x) \mid=\infty\}$ is a null set.

(i) and (ii) suggest to identify a.e. equal functions in $\mathscr{L}^{1}$. If we do so, we can now regard $\mathscr{L}^{1}$ as a real vector space since it follows from (iii) and (ii) that, given any $f \in \mathscr{L}^{1}$, we can find a real valued $g \in \mathscr{L}^{1}$ with $f=g$ a.e. The $(I-)$ integral $\int d I$ is then easily recognized as a positive, linear functional on $\mathscr{L}^{1}$.

Moreover \|\| is a pseudonorm on $\mathscr{L}^{1}$, i.e. it has the norm properties but $+\infty$ is not excluded as a possible value. Defining Cauchy sequences and completeness as usual, we mention that $\left(\mathscr{L}^{1},\|\|\right)$ is a complete, pseudonormed vector space.

If the condition

$$
\bar{I}(f)=\int f d I \quad \forall f \in\left(\mathscr{L}^{1}\right)^{+}
$$

is satisfied (which need not be true in general, cf. [1]), the monotone convergence theorem (and some other results) remain valid.

\section{The Diagonal DeRIVATIVE AND THE $I_{d}$-INTEGRAL}

Let us first put together some other prerequisites. For the following, let $J \subset \mathbf{R}$ be any interval (bounded or not). The Dini derivatives $D^{+} F, D^{-} F, D_{+} F$, 
$D_{-} F$ of a function $F: J \rightarrow \mathbf{R}$ are defined as usual. For $F, G: J \rightarrow \mathbf{R}$ the four inequalities

$$
\begin{aligned}
& D^{ \pm}(F+G)(x) \leq D^{ \pm} F(x)+D^{ \pm} G(x), \\
& D_{ \pm}(F+G)(x) \geq D_{ \pm} F(x)+D_{ \pm} G(x)
\end{aligned}
$$

hold respectively, whenever the right-hand sums are defined in $\overline{\mathbf{R}}$. If $G$ is differentiable in $x$ (with derivative in $\mathbf{R}$ ) we have

$$
\begin{aligned}
& D^{ \pm}(F+G)(x)=D^{ \pm} F(x)+G^{\prime}(x), \\
& D_{ \pm}(F+G)(x)=D_{ \pm} F(x)+G^{\prime}(x) .
\end{aligned}
$$

For the sake of simplicity, we say a property $Q=Q(x)$ for elements $x \in X$ holds nearly everywhere (n.e.), if the set $\{x \in X \mid Q(x)$ is false $\}$ is at most countable.

2. Proposition. For any $F: J \rightarrow \mathbf{R}$ one has $D_{-} F \leq D^{+} F$ n.e.

A simple proof is given in [4, Chapter IV, 4].

3. Proposition. For any continuous $F: J \rightarrow \mathbf{R}$ :

$$
D^{+} F \geq 0 \text { n.e. } \Rightarrow F \text { is nondecreasing. }
$$

Again, a simple proof can be found in [4, Chapter XI, 4].

Let $c \in[-\infty, \infty]$ be an endpoint of the interval $J \subset \mathbf{R}$ and $F: J \rightarrow \mathbf{R}$ a real function. We say that $F(c)$ exists, if the limit

$$
F(c):=\lim _{\substack{x \in J \\ x \rightarrow c}} F(x) \in \mathbf{R}
$$

exists. Of course, this is always true if $c \in J$ and $F$ is continuous in $c$. For an interval $J$ with endpoints $a \leq b$ in $\overline{\mathbf{R}}$ we write $J=\langle a, b\rangle$ if we want to leave it open which of the endpoints $a, b$ do or do not belong to $J$.

For an interval $J=\langle a, b\rangle$ we define $\mathscr{H}=\mathscr{H}(J)$ to be the space of all continuous functions $F: J \rightarrow \mathbf{R}$ for which $F(a), F(b)$ exist and which satisfy the condition

$$
D_{-} F=D^{+} F \in \mathbf{R} \text { n.e. }
$$

4. Proposition. For $F, G \in \mathscr{H}$ and $c \geq 0$ we have $c F, F+G \in \mathscr{H}$ and

$$
\begin{gathered}
D_{-}(c F)=c D_{-} F=c D^{+} F=D^{+}(c F) \text { n.e. } \\
D_{-}(F+G)=D_{-} F+D_{-} G=D^{+} F+D^{+} G=D^{+}(F+G) \text { n.e. }
\end{gathered}
$$

Proof. The assertion for $c F$ is trivial. For $F, G \in \mathscr{H}$ we have

$$
\begin{aligned}
& D^{+}(F+G) \leq D^{+} F+D^{+} G \text { n.e. and } \\
& D_{-}(F+G) \geq D_{-} F+D_{-} G \text { n.e. }
\end{aligned}
$$

since the right-hand Dini derivatives are in $\mathbf{R}$ n.e. and thus the right-hand sums are defined n.e. By proposition 2, applied to $F+G$, we have

$$
D_{-} F+D_{-} G \leq D_{-}(F+G) \leq D^{+}(F+G) \leq D^{+} F+D^{+} G \text { n.e. }
$$

Since $D_{-} F=D^{+} F \in \mathbf{R}$ and $D_{-} G=D^{+} G \in \mathbf{R}$ n.e., equality must hold n.e. in $(*)$ and the assertion follows. 
We now define $\mathscr{G}=\mathscr{G}(J)$ to be

$$
\mathscr{G}:=\mathscr{H}-\mathscr{H}=\left\{F_{1}-F_{2} \mid F_{1}, F_{2} \in \mathscr{H}\right\}
$$

and call the members of $\mathscr{G}$ diagonally differentiable ( $d$-differentiable) functions. For $F=F_{1}-F_{2} \in \mathscr{G}\left(F_{1}, F_{2} \in \mathscr{H}\right)$ we define a function $\widehat{D}\left(F_{1}, F_{2}\right)$ on $J$ by

$$
\widehat{D}\left(F_{1}, F_{2}\right)(x):=\left\{\begin{array}{l}
D^{+} F_{1}(x)-D^{+} F_{2}(x) \text { if } D^{+} F_{1}(x), D^{+} F_{2}(x) \in \mathbf{R}, \\
0 \quad \text { elsewhere }
\end{array}\right.
$$

and call $\widehat{D}\left(F_{1}, F_{2}\right)$ a diagonal derivative (d-derivative) of $F$. Note that

$$
\widehat{D}\left(F_{1}, F_{2}\right)=D^{+} F_{1}-D^{+} F_{2} \text { n.e. }
$$

by the definition of $\mathscr{H}$. It makes no sense to define a pointwise diagonal derivative, because it is not too difficult to find a function $F \in \mathscr{G}$ having decompositions $F=F_{1}-F_{2}=G_{1}-G_{2}\left(F_{1}, F_{2}, G_{1}, G_{2} \in \mathscr{H}\right)$, such that

$$
D_{-} F_{i}=D^{+} F_{i} \in \mathbf{R}, \quad D_{-} G_{i}=D^{+} G_{i} \in \mathbf{R} \text { everywhere }(i=1,2)
$$

(except, perhaps, at the endpoints of $J$ ), but

$$
D^{+} F_{1}(x)-D^{+} F_{2}(x) \neq D^{+} G_{1}(x)-D^{+} G_{2}(x) \quad \forall x \in A,
$$

with a countable set $A \subset J$; moreover, such functions exist for every countable $A$, provided that $A$ has no accumulation-point in the interior of $J$. But this is almost the worst thing that can happen:

5. Lemma. Two diagonal derivatives of a function $F \in \mathscr{G}$ coincide n.e.

Proof. Let $F=F_{1}-F_{2}=G_{1}-G_{2}\left(F_{1}, F_{2}, G_{1}, G_{2} \in \mathscr{H}\right)$. By Proposition 4 we have n.e.

$$
D^{+} F_{1}+D^{+} G_{2}=D^{+}\left(F_{1}+G_{2}\right)=D^{+}\left(F_{2}+G_{1}\right)=D^{+} F_{2}+D^{+} G_{1} .
$$

By the definition of $\mathscr{H}$, all Dini derivatives are in $\mathbf{R}$ n.e., thus

$$
\widehat{D}\left(F_{1}, F_{2}\right)=D^{+} F_{1}-D^{+} F_{2}=D^{+} G_{1}-D^{+} G_{2}=\widehat{D}\left(G_{1}, G_{2}\right) \text { n.e. }
$$

6. Proposition. Let $F \in \mathscr{G}, F=F_{1}-F_{2}\left(F_{1}, F_{2} \in \mathscr{H}\right)$.

(i) $\widehat{D}\left(F_{1}, F_{2}\right) \geq 0$ n.e. $\Rightarrow F$ is nondecreasing.

(ii) $\widehat{D}\left(F_{1}, F_{2}\right)=0$ n.e. $\Rightarrow F=$ constant.

Proof. Since $D^{+} F_{1}, D^{+} F_{2} \in \mathbf{R}$ n.e. we conclude

$$
\begin{aligned}
& D^{+} F_{1}=D^{+}\left(F+F_{2}\right) \leq D^{+} F+D^{+} F_{2} \text { n.e. } \Rightarrow \\
& D^{+} F \geq D^{+} F_{1}-D^{+} F_{2}=\widehat{D}\left(F_{1}, F_{2}\right) \geq 0 \text { n.e. }
\end{aligned}
$$

Thus, if $\widehat{D}\left(F_{1}, F_{2}\right) \geq 0$ n.e. we must have $D^{+} F \geq 0$ n.e. and (i) follows from Proposition 3. Of course, (ii) is any easy consequence of (i).

Lemma 5 allows us to speak of the diagonal derivative $\widehat{D} F$ of a function $F \in \mathscr{G}$, if we identify diagonal derivatives which are equal n.e. In this sense, $\widehat{D}$ is easily recognized to operate linearly on $\mathscr{G}$ (use Proposition 4). Proposition 6 ensures that any $F \in \mathscr{G}$ is (up to a constant) uniquely determinated by its diagonal derivative $\widehat{D} F$. In the remainder, for $F \in \mathscr{G}$, the symbol $\widehat{D} F$ stands for any diagonal derivative $\widehat{D}\left(F_{1}, F_{2}\right) \quad\left(F_{1}, F_{2} \in \mathscr{H}, F=F_{1}-F_{2}\right)$. 
7. Lemma. If $F \in \mathscr{G}$ and $M=\left\{x \in J \mid F^{\prime}(x) \in \mathbf{R}\right.$ exists $\}$, then

$$
\widehat{D} F=F^{\prime} \text { n.e. on } M \text {. }
$$

Proof. With $F=F_{1}-F_{2} \quad\left(F_{1}, F_{2} \in \mathscr{H}\right)$ we have

$$
\begin{aligned}
D^{+} F_{1} & =D^{+}\left(F+F_{2}\right)=F^{\prime}+D^{+} F_{2} \text { everywhere on } M \Rightarrow \\
F^{\prime} & =D^{+} F_{1}-D^{+} F_{2}=\widehat{D}\left(F_{1}, F_{2}\right)=\widehat{D} F \text { n.e. on } M .
\end{aligned}
$$

8. Definition. A function $F \in \mathscr{G}$ is called an indefinite diagonal integral (indefinite $d$-integral) of $f: J=\langle a, b\rangle \rightarrow \mathbf{R}$, if $f=\widehat{D} F$ n.e. We furthermore define

$$
\mathscr{E}_{d}=\mathscr{E}_{d}(J):=\{f: J \rightarrow \mathbf{R} \mid f \text { has an indefinite } d \text {-integral }\} .
$$

For $f \in \mathscr{E}_{d}$ and an indefinite $d$-integral $F$ of $f$ we set

$$
I_{d}(f):=F(b)-F(a) .
$$

Proposition 6(ii) ensures, that $I_{d}$ is well defined. Obviously, $\mathscr{E}_{d}$ is a real vector space and $I_{d}: \mathscr{E}_{d} \rightarrow \mathbf{R}$ is a linear and, by Proposition 6(i), also a positive functional. We want to show, that $I_{d}$ is a Daniell integral, but for the missing property of continuity from below we need some further preparations. By $\mathscr{C}_{c}=$ $\mathscr{C}_{c}(J)$ we denote the space of all continuous $g: J \rightarrow \mathbf{R}$ with compact support (relative to $J$ ).

9. Lemma. Let $J=\langle a, b\rangle$ and $g_{i} \in \mathscr{C}_{c}^{+}(i=0,1,2, \ldots)$ with

$$
\sum_{0}^{\infty} \int_{a}^{b} g_{i}(t) d t<\infty
$$

If $g=\sum g_{i}$ and $G: J \rightarrow \mathbf{R}$ is defined by

$$
G(x):=\int_{a}^{x} g(t) d t
$$

then $g \leq D_{-} G$ everywhere on $J \backslash\{a\}$.

Proof. Let $x \in J \backslash\{a\}$. For arbitrary $y \in(a, x)$ and $n \in \mathbf{N}$ we can estimate

$$
\frac{G(x)-G(y)}{x-y} \geq \frac{1}{x-y} \sum_{i=0}^{n} \int_{y}^{x} g_{i}(t) d t .
$$

Since the right-hand side tends to $\sum_{i=0}^{n} g_{i}(x)$ as $y \uparrow x$, it follows that

$$
D_{-} G(x) \geq \sum_{i=0}^{n} g_{i}(x) \quad \forall n \in \mathbf{N} \Rightarrow D_{-} G(x) \geq g(x) .
$$

10. Lemma. Let $f \in \mathscr{E}_{d}$ and $g_{i}$ be nonnegative, Lebesgue integrable functions $(i=0,1,2, \ldots)$. Then we have

$$
f \leq \sum_{i=0}^{\infty} g_{i} \text { n.e. } \Rightarrow I_{d}(f) \leq \sum_{i=0}^{\infty} \int_{a}^{b} g_{i}(t) d t .
$$

Proof. We may assume

$$
\sum_{i=0}^{\infty} \int_{a}^{b} g_{i}(t) d t<\infty
$$


for otherwise there is nothing to show. Let us first regard the case, where all $g_{i}$ are in $\mathscr{C}_{c}^{+}$. Building $G$ as in Lemma 9, from the assumption $f \leq \sum g_{i}$ n.e. it follows

$$
0 \leq \sum_{i=0}^{\infty} g_{i}-f \leq D_{-} G-f \text { n.e. }
$$

by Lemma 9. If $F=F_{1}-F_{2} \quad\left(F_{1}, F_{2} \in \mathscr{H}\right)$ is an indefinite $d$-integral of $f \in \mathscr{E}_{d}$ we therefore obtain n.e.

$$
\begin{aligned}
0 & \leq D_{-} G-D^{+} F_{1}+D^{+} F_{2} \\
& =D_{-} G+D_{-} F_{2}-D^{+} F_{1} \\
& \leq D_{-}\left(G+F_{2}\right)-D^{+} F_{1} \\
& \leq D^{+}\left(G+F_{2}\right)-D^{+} F_{1} \quad(\text { by Proposition } 2) \\
& =D^{+}\left(G-F+F_{1}\right)-D^{+} F_{1} \\
& \leq D^{+}(G-F)+D^{+} F_{1}-D^{+} F_{1}=D^{+}(G-F) .
\end{aligned}
$$

Thus $D^{+}(G-F) \geq 0$ n.e. and $G-F$ is nondecreasing by Proposition 3. This yields

as desired.

$$
I_{d}(f)=F(b)-F(a) \leq G(b)-G(a)=\sum_{i=0}^{\infty} \int_{a}^{b} g_{i}(t) d t,
$$

If now the $g_{i}$ are arbitrary, nonnegative, Lebesgue integrable functions, for a given $\varepsilon>0$ we can find $g_{i k} \in \mathscr{C}_{c}^{+}(i, k=0,1,2, \ldots)$ such that for every $i$ the following is satisfied:

$$
\left|g_{i}-g_{i 0}\right| \leq \sum_{k=1}^{\infty} g_{i k}, \quad\left|\int g_{i}-\int g_{i 0}\right|<\frac{\varepsilon}{2^{i}}, \quad \sum_{k=1}^{\infty} \int g_{i k}<\frac{\varepsilon}{2^{i}} .
$$

This is possible by the definition of the Lebesgue integral with the method of Daniell. Thus, if $f \leq \sum_{i=0}^{\infty} g_{i}$ n.e., we have $f \leq \sum_{i, k=0}^{\infty} g_{i k}$ n.e. a fortiori and hence

$$
I_{d}(f) \leq \sum_{i=0}^{\infty}\left(\int g_{i 0}+\sum_{k=1}^{\infty} \int g_{i k}\right)<\sum_{i=0}^{\infty}\left(\int g_{i}+\frac{\varepsilon}{2^{i}}+\frac{\varepsilon}{2^{i}}\right)=\sum_{i=0}^{\infty} \int g_{i}+4 \varepsilon
$$

by what has already been shown. Since $\varepsilon>0$ was arbitrary, the general assertion follows.

From now on the term "almost everywhere" (a.e.) is used in the ordinary Lebesgue sense.

11. Proposition. Every $f \in \mathscr{E}_{d}^{+}$is Lebesgue integrable with

$$
I_{d}(f)=\int_{a}^{b} f(t) d t
$$

Proof. With an indefinite $d$-integral $F$ of $f$ we have

$$
\widehat{D} F=f \geq 0 \text { n.e., }
$$

forcing $F$ to be nondecreasing by Proposition 6(i). A result of Lebesgue yields that $F$ is differentiable a.e. and $F^{\prime}$ is Lebesgue integrable with

$$
\int_{a}^{b} F^{\prime}(t) d t \leq F(b)-F(a) .
$$


Using Lemma (7) we obtain $f=F^{\prime}$ a.e., hence $f$ is Lebesgue integrable with

$$
\int_{a}^{b} f(t) d t=\int_{a}^{b} F^{\prime}(t) d t \leq F(b)-F(a)=I_{d}(f) .
$$

On the other hand, since we know that $f$ is Lebesgue integrable, we also get

$$
I_{d}(f) \leq \int_{a}^{b} f(t) d t
$$

as a special application of Lemma 10 .

For $f \in \mathscr{E}_{d}, f_{n} \in \mathscr{E}_{d}^{+}$with $\sum_{1}^{\infty} f_{n} \geq f$, the combination of Lemma 10 and Proposition 11 shows

$$
I_{d}(f) \leq \sum_{1}^{\infty} \int_{a}^{b} f_{n}(t) d t=\sum_{1}^{\infty} I_{d}\left(f_{n}\right) .
$$

Thus $I_{d}$ is continuous from below and we have shown:

12. Theorem. The functional $I_{d}: \mathscr{E}_{d} \rightarrow \mathbf{R}$ is a Daniell integral.

Notice that obviously $\mathscr{C}_{c} \subset \mathscr{E}_{d}$ and that an ordinary indefinite integral of $g \in \mathscr{C}_{c}$ is also an indefinite $d$-integral of $g$, briefly:

$$
\mathscr{C}_{c} \subset \mathscr{C}_{d}, I_{d} \mid \mathscr{C}_{c}=\text { Riemann or Lebesgue integral. }
$$

As described in $\S 1$, we now obtain the functional $\bar{I}_{d}$ and a norm \|\|$_{d}$ defined by $\|f\|_{d}=\bar{I}_{d}(|f|)$ for an arbitrary $f: J \rightarrow \overline{\mathbf{R}}$. We also get the space $\mathscr{L}_{d}^{1}=\mathscr{L}_{d}^{1}\left(J, \mathscr{E}_{d}, I_{d}\right)$ of the $I_{d}$-integrable functions, together with the $I_{d^{-}}$ integral $\int d I_{d}$ on $\mathscr{L}_{d}^{1}$. With the ordinary Lebesgue norm \|\| , defined by

$$
\|f\|:=\inf \left\{\sum_{1}^{\infty} \int g_{n}\left|g_{n} \in \mathscr{C}_{c}^{+}, \sum_{1}^{\infty} g_{n} \geq\right| f \mid\right\}
$$

for any $f: J \rightarrow \overline{\mathbf{R}}$, we see at once, that \|\|$_{d} \leq\|\|$ as a consequence of the foregoing remark. To see that the reverse inequality is also true, let $f: J \rightarrow \overline{\mathbf{R}}$ be arbitrary. For showing $\|f\| \leq\|f\|_{d}$ we may assume $\|f\|_{d}<\infty$. Then, for $\varepsilon>0$, we find $g_{n} \in \mathscr{E}_{d}^{+}$fulfilling

$$
|f| \leq \sum_{1}^{\infty} g_{n}, \quad \sum_{1}^{\infty} I_{d}\left(g_{n}\right)<\|f\|_{d}+\varepsilon .
$$

By Proposition 11, the countable subadditivity of the Lebesgue norm and the fact that

$$
\int_{a}^{b}|h(t)| d t=\|h\|
$$

for Lebesgue integrable $h$, we can estimate

$$
\|f\| \leq\left\|\sum_{1}^{\infty} g_{n}\right\| \leq \sum_{1}^{\infty}\left\|g_{n}\right\|=\sum_{1}^{\infty} \int_{a}^{b} g_{n}(t) d t=\sum_{1}^{\infty} I_{d}\left(g_{n}\right)<\|f\|_{d}+\varepsilon .
$$

This yields the desired inequality since $\varepsilon>0$ was arbitrary. Thus we have proved \|\|$_{d}=\|\|$, an important fact to remember for the remainder. 
13. Proposition. Every Lebesgue integrable function is $I_{d}$-integrable and the integrals coincide.

Proof. For any Lebesgue integrable $f$ we find $g_{n} \in \mathscr{C}_{c}$ with $\left\|f-g_{n}\right\| \rightarrow 0$. Since $\mathscr{C}_{c} \subset \mathscr{E}_{d}$ and \|\|$=\|\|_{d}$ this shows at once that $f \in \mathscr{L}_{d}^{1}$. Furthermore we have

$$
\int_{a}^{b} f(t) d t=\lim _{n \rightarrow \infty} \int_{a}^{b} g_{n}(t) d t=\lim _{n \rightarrow \infty} I_{d}\left(g_{n}\right)=\int_{a}^{b} f d I_{d} .
$$

14. Proposition. For all $f \in \mathscr{L}_{d}^{1}$ we have:

(i) $f$ is Lebesgue measurable.

(ii) $f$ is Lebesgue integrable $\Leftrightarrow\|f\|<\infty$.

Proof. To prove (i), choose $g_{n} \in \mathscr{E}_{d}$ with $\left\|f-g_{n}\right\|_{d}=\left\|f-g_{n}\right\| \rightarrow 0$. It is well known that in this case we can find a subsequence $g_{n_{k}}$ with $g_{n_{k}} \rightarrow f$ a.e. as $k \rightarrow \infty$. Thus, being a.e. the limit of the $g_{n_{k}}, f$ surely is Lebesgue measurable if the $g_{n_{k}}$ are. But this is true, because every $g \in \mathscr{E}_{d}$ is n.e. the difference of two Dini derivatives of continuous functions and the latter are well known to be even Borel measurable. This proves (i), and (ii) follows, because the statement of (ii) is true for every Lebesgue measurable $f$.

15. Corollary. (i) The space $\left(\mathscr{L}_{d}^{1}\right)^{+}$consists exactly of the nonnegative, Lebesgue integrable functions and the integrals coincide.

(ii) We have

$$
\bar{I}_{d}(f)=\int f d I_{d} \quad \forall f \in\left(\mathscr{L}_{d}^{1}\right)^{+} .
$$

We already mentioned the importance of (ii) at the end of $\S 1$.

Proof. With a look at Proposition 13, for proving (i), we only have to show, that every $f \in\left(\mathscr{L}_{d}^{1}\right)^{+}$is Lebesgue integrable. Since $f$ is Lebesgue measurable by (i) of the last proposition, the functions

$$
f_{n}:=\left(f \chi_{J \cap[-n, n]}\right) \wedge n
$$

are Lebesgue integrable with $f_{n} \uparrow f$. But now, with the aid of Proposition 13 and the positivity of $\int d I_{d}$, we have

$$
\int_{a}^{b} f_{n}(t) d t=\int_{a}^{b} f_{n} d I_{d} \leq \int_{a}^{b} f d I_{d} \quad \forall n .
$$

The monotone convergence theorem yields that $f=\lim f_{n}$ is Lebesgue integrable, (i) is proved.

Now (ii) follows immediately since for $f \in\left(\mathscr{L}_{d}^{1}\right)^{+}$we get

$$
\bar{I}_{d}(f)=\|f\|_{d}=\|f\|=\int_{a}^{b} f(t) d t=\int_{a}^{b} f d I_{d} .
$$

16. Corollary. We have $f \in \mathscr{L}_{d}^{1}$ if and only if $f$ admits a representation

$$
f=g+h \quad g \in \mathscr{E}_{d}, h \text { Lebesgue integrable. }
$$

In this case, for an arbitrary $\varepsilon>0$ we can achieve $\|h\|<\varepsilon$.

Proof. Of course, every $f$ decomposable in the indicated manner is in $\mathscr{L}_{d}^{1}$ (consider Proposition 13). Conversely, let $f \in \mathscr{L}_{d}^{1}$ and $\varepsilon>0$. We then find 
$g \in \mathscr{E}_{d}$ with $\|f-g\|_{d}=\|f-g\|<\varepsilon$. Now $f=g+h$ with $h:=f-g$ is the desired representation, because $\|h\|<\varepsilon$ yields the Lebesgue integrability of $h$ by proposition $14(\mathrm{ii})$.

The preceding corollary shall play an important role for our main purpose. From it we recognize that indefinite $I_{d}$-integrals are exactly the sums of $d$ differentiable and absolutely continuous functions. In the following section, we shall show that decomposability into the sum of a $d$-differentiable and an absolutely continuous function is equivalent to the ACG-property. From this, it is a short step to realize, that $\mathscr{L}_{d}^{1}$ consists exactly of the Denjoy integrable functions.

\section{A NEW APPROACH TO THE GENERAL DENJOY INTEGRAL}

For sake of simplicity, from now on let $J=[a, b]$ be a compact interval. This is not at all essential, but customary in literature when dealing with the Denjoy integral. The reader is assumed to be familiar with absolutely continuous functions (AC-functions), generalized absolutely continuous functions (ACG-functions) and the notion of the (general) Denjoy integral. For a detailed decription of these tools we refer to [3].

\section{Proposition. If a continuous $F: J \rightarrow \mathbf{R}$ satisfies the conditions}

$$
-\infty<D_{-} \text {Fn.e. and } D^{+} F<\infty \text { n.e. }
$$

then $F$ is ACG on $J$.

Proof. For a suitable, at most countable $A \subset J$ and every $x \in J \backslash A$ we find $n \in \mathbf{N}$, such that for $t \in J$ we have

$$
\begin{aligned}
& 0 \leq t-x \leq \frac{1}{n} \Rightarrow F(t)-F(x) \leq n(t-x), \\
& 0 \leq x-t \leq \frac{1}{n} \Rightarrow F(x)-F(t) \geq-n(x-t) .
\end{aligned}
$$

Therefore, letting

$$
M_{n}:=\left\{x \in J|\forall t \in J:| x-t\left|\leq \frac{1}{n} \Rightarrow F(t)-F(x) \leq n\right| t-x \mid\right\},
$$

we have the countable union

$$
J=\bigcup_{1}^{\infty} M_{n} \cup \bigcup_{a \in A}\{a\} .
$$

Thus it suffices to show that $F$ is ACG on every $M_{n}$. But if we set

$$
M_{n}^{i}:=M_{n} \cap\left[\frac{i}{n}, \frac{i+1}{n}\right] \quad(i \in \mathbf{Z}),
$$

$M_{n}$ is the countable union of the $M_{n}^{i}$ and $F$ is AC on each $M_{n}^{i}$. For $x, y \in$ $M_{n}^{i}$ we have $F(x)-F(y) \leq n|x-y|$ and hence $|F(x)-F(y)| \leq n|x-y|$ since the roles of $x$ and $y$ can be interchanged. From this, the absolute continuity of $F$ on $M_{n}^{i}$ follows immediately.

We now want to establish an important relation between ACG-functions and the members of $\mathscr{G}$. For an ACG-function $F$ we denote by $F_{a p}^{\prime}$ its (a.e. defined) approximate derivative (cf. [3]). 
18. Theorem. Let $F \in \mathscr{G}$.

(i) $F$ is $A C G$ on $[a, b]$ and hence approximately differentiable a.e.

(ii) We have $\widehat{D} F=F_{a p}^{\prime}$ a.e.

Proof. By Proposition 17, the members of $\mathscr{H}$ are obviously ACG on $J$ and hence so are the members of $\mathscr{G}=\mathscr{H}-\mathscr{H}$.

Now let $F=F_{1}-F_{2} \quad\left(F_{1}, F_{2} \in \mathscr{H}\right)$ and define

$$
\begin{aligned}
M:=\left\{x \in J \mid\left(F_{i}\right)_{a p}^{\prime}(x) \text { exists, } D_{-} F_{i}(x)=D^{+} F_{i}(x)\right. & \in \mathbf{R}(i=1,2), \\
\widehat{D}\left(F_{1}, F_{2}\right)(x) & \left.=D^{+} F_{1}(x)-D^{+} F_{2}(x)\right\} .
\end{aligned}
$$

Because all constituting conditions for $M$ are satisfied a.e. or n.e., for proving (ii) it suffices to show that $F_{a p}^{\prime}(x)=\widehat{D}\left(F_{1}, F_{2}\right)(x)$ for $x \in M$. Therefore, let $x \in M$ and $\mu_{i}:=\left(F_{i}\right)_{a p}^{\prime}(x) \quad(i=1,2)$. Then, by the definition of the approximate derivative, given any $\varepsilon>0, x$ is a point of density of the sets

$$
A_{i}(\varepsilon):=\left\{y \in J \backslash\{x\} \mid \mu_{i}-\varepsilon \leq \frac{F_{i}(y)-F_{i}(x)}{y-x} \leq \mu_{i}+\varepsilon\right\} \quad(i=1,2)
$$

and so, a fortiori, $x$ must be a bilateral accumulation point of these sets. From this fact we conclude $D_{-} F_{i}(x) \leq \mu_{i}+\varepsilon$ and $D^{+} F_{i}(x) \geq \mu_{i}-\varepsilon$ for arbitrary $\varepsilon>0$, thus

$$
\mu_{i} \leq D^{+} F_{i}(x)=D_{-} F_{i}(x) \leq \mu_{i}
$$

and equality must hold $(i=1,2)$. So, for $x \in M$

$$
\begin{aligned}
F_{a p}^{\prime}(x) & =\left(F_{1}\right)_{a p}^{\prime}(x)-\left(F_{2}\right)_{a p}^{\prime}(x) \\
& =\mu_{1}-\mu_{2}=D^{+} F_{1}(x)-D^{+} F_{2}(x)=\widehat{D}\left(F_{1}, F_{2}\right)(x),
\end{aligned}
$$

what we wanted to show.

We now define a property for functions $F:[a, b] \rightarrow \mathbf{R}$, which turns out to be equivalent to the ACG property.

19. Definition. A function $F:[a, b] \rightarrow \mathbf{R}$ is called decomposable over $[a, b]$, if there exist $G_{1}, G_{2} \in \mathscr{H}$ and an absolutely continuous $H$ such that

$$
F=G_{1}-G_{2}+H
$$

The decomposition $(*)$ is called an $\varepsilon$-decomposition $(\varepsilon>0)$, if the following conditions are satisfied:

(i) $G_{2}(a)=G_{2}(b)=0,\left\|G_{2}\right\|_{\infty}<\varepsilon$.

(ii) $H(a)=H(b)=0,\left\|H^{\prime}\right\|<\varepsilon$ (and hence $\|H\|_{\infty}<\varepsilon$ ).

(iii) $\left\|G_{1}\right\|_{\infty},\left\|G_{2}\right\|_{\infty} \leq 2\|F\|_{\infty}$.

Here \|\|$_{\infty}$ denotes the supremum norm and \|| $\|$ the Lebesgue integral norm.

20. Lemma. For every decomposable $F$ and every $\varepsilon>0$ there exists an $\varepsilon$ decomposition of $F$.

Proof. (Sketch) Let $F=U_{1}-U_{2}+S \quad\left(U_{1}, U_{2} \in \mathscr{H}, S\right.$ absolutely continuous) be any decomposition of $F$. If we change $S$ by a suitable linear function (which we add to $U_{1}$ ), we may assume $S(a)=S(b)=0$. Since $S$ is absolutely continuous, $S^{\prime}$ is Lebesgue integrable and to $\varepsilon>0$ we find a continuous $g$ 
with $\left\|S^{\prime}-g\right\|<\varepsilon$. If $G$ is an indefinite integral of $g$, we may arrange it so that $G(a)=G(b)=0$. Thus, setting

$$
G_{1}:=U_{1}+G, \quad G_{2}:=U_{2}, \quad H:=S-G,
$$

we may assume that (ii) holds.

Now, if $W$ is a suitable, piecewise linear interpolation of $G_{2}$ fulfilling $W(a)=G_{2}(a), W(b)=G_{2}(b)$, we can replace $G_{2}$ be $G_{2}-W$ and $G_{1}$ by $G_{1}-W$, hence verifying (i) and (iii).

Every decomposable function is continuous since absolutely continuous functions and functions in $\mathscr{H}$ are continuous. We call $F$ decomposable over $x \in[a, b]$, if there exists $\delta>0$, such that $F$ is decomposable over $[a, b] \cap$ $[x-\delta, x+\delta]$. Evidently, if $F$ is decomposable over $J, F$ is decomposable over every compact subinterval of $J$.

21. Lemma. Let $F:[a, b] \rightarrow \mathbf{R}$ be a real function.

(i) If $a<c<b$ and $F$ is decomposable over $[a, c]$ and $[c, b]$, then $F$ is decomposable over $[a, b]$.

(ii) If $F$ is continuous and decomposable over every $[a, \beta]$ with $a \leq \beta<b$ (over every $[\alpha, b]$ with $a<\alpha \leq b$ ), then $F$ is decomposable over $[a, b]$.

(iii) If $F$ is decomposable over every $x \in[a, b]$, then $F$ is decomposable over $[a, b]$.

Proof. The assertions (i) and (iii) are more or less trivial.

To prove (ii), let $F$ be decomposable over every $[a, \beta]$ with $a \leq \beta<b$ and choose real $\beta_{i}(i=0,1,2, \ldots)$ with

$$
a=\beta_{0}<\beta_{1}<\beta_{2}<\cdots, \quad \beta_{i} \uparrow b .
$$

By hypothesis, $F$ is decomposable over every $\left[\beta_{i-1}, \beta_{i}\right]$. For every $i$ we find a $1 / 2^{i}$-decomposition of $F$ over $\left[\beta_{i-1}, \beta_{i}\right]$ :

$$
F \mid\left[\beta_{i-1}, \beta_{i}\right]=G_{1 i}-G_{2 i}+H_{i} \quad(i=0,1,2, \ldots) .
$$

Let $G_{1}:[a, b] \rightarrow \mathbf{R}$ be the function with $G_{1} \mid\left[\beta_{i-1}, \beta_{i}\right]=G_{1 i}$ for $i=0,1,2, \ldots$ $\left(G_{1}(b):=F(b)\right)$ and define $G_{2}$ and $H$ analogously. Now it is not difficult to verify that $F=G_{1}-G_{2}+H$ is a decomposition of $F$ over $[a, b]$.

22. Lemma. Let $F:[a, b] \rightarrow \mathbf{R}$ be continuous with $F(a)=F(b)=0$. Then there exists a continuous, n.e. differentiable $W:[a, b] \rightarrow \mathbf{R}$ fulfilling

$$
W \geq|F|, \quad\|W\|_{\infty}=\|F\|_{\infty}, \quad W(a)=W(b)=0 .
$$

Instead of the elementary proof we just say that a suitable, piecewise linear $W$ does what we want, if, perhaps, the (possibly countably many) points of nondifferentiability of $W$ accumulate in $a$ or $b$. Now we are in the position to prove our main result.

23. Theorem. A function $F:[a, b] \rightarrow \mathbf{R}$ is $A C G$ on $[a, b]$ if and only if $F$ is decomposable over $[a, b]$.

Proof. Absolutely continuous functions and, by Theorem 18(i), members of $\mathscr{H}$ are ACG on $[a, b]$ and hence so is every decomposable $F$. 
The converse assertion is much more subtle. First, let $F$ be ACG on $[a, b]$ and let

$$
P:=\{x \in[a, b] \mid F \text { is not decomposable over } x\} .
$$

It is obvious that $P$ is closed. To see that $P$ contains no isolated points, assume that $c \in P$ is such a point. Then there exists a suitable $\delta>0$ such that $F$ is decomposable over every $x \in[c-\delta, c+\delta] \backslash\{c\}$. Lemma 21(iii) yields the decomposability of $F$ over $[c-\delta, u]$ and $[v, c+\delta]$ whenever we have $c-\delta \leq u<c<v \leq c+\delta$. Using (ii) and (i) of Lemma 21, we obtain the decomposability of $F$ over $[c-\delta, c+\delta]$, contradicting $c \in P$. Thus $P$ is closed and contains no isolated points.

By Lemma 21(iii) it suffices to show $P=\varnothing$, so we will disprove $P \neq \varnothing$. But, assuming $P \neq \varnothing$, by a well-known theorem (cf. [3, Chapter VII, §9]) we find $\alpha<\beta$ in $\mathbf{R}$, fulfilling $(\alpha, \beta) \cap P \neq \varnothing$ and $F$ is AC on $(\alpha, \beta) \cap P$. Since $P$ contains no isolated points and $(\alpha, \beta) \cap P \neq \varnothing$, we find $a_{0}, b_{0} \in(\alpha, \beta) \cap P$ such that $F$ is AC on $P_{0}:=\left[a_{0}, b_{0}\right] \cap P$ and $P_{0}$ contains infinitely many points. We shall show, that $F$ is decomposable over $\left[a_{0}, b_{0}\right]$, because then $F$ would be decomposable over a point $c \in\left(a_{0}, b_{0}\right) \cap P$ (which is nonvoid since $P_{0}$ contains infinitely many points), contradicting $c \in P$. Therefore, to show that $F$ is decomposable over $\left[a_{0}, b_{0}\right]$, let $\left\{J_{k} \mid k=1,2, \ldots\right\}$ be the (at most countable) family of closed intervals contiguous to $P_{0}$ in $\left[a_{0}, b_{0}\right]$, i.e. the closures of the components of $\left[a_{0}, b_{0}\right] \backslash P_{0}$. Define a function $F_{1}$ on $\left[a_{0}, b_{0}\right]$ by

$$
F_{1}:=\left\{\begin{array}{l}
F \text { on } P_{0} \\
\text { linearly interpolated on the } J_{k} .
\end{array}\right.
$$

The latter means: If $J_{k}=\left[a_{k}, b_{k}\right]$, the graph of $F_{1}$ over $J_{k}$ should connect linearly the points $\left(a_{k}, F\left(a_{k}\right)\right)$ and $\left(b_{k}, F\left(b_{k}\right)\right)$. Using that $F$ is $\mathrm{AC}$ on $P_{0}$, the reader should have no difficulties to check, that $F_{1}$ is absolutely continuous on the whole of $\left[a_{0}, b_{0}\right]$. Therefore, if we could show that $\widetilde{F}:=F-F_{1}$ is decomposable over $\left[a_{0}, b_{0}\right]$, so is $F$ and the proof would be complete.

For this purpose, let us put together the properties of $\widetilde{F}$. As a difference of continuous functions, $\widetilde{F}$ itself is continuous. By construction, $\widetilde{F} \mid P_{0}=0 . F$ is decomposable over every $J_{k}$, as an easy argumentation (using Lemma 21(iii) and (ii)) shows. Hence $\widetilde{F}$ is also decomposable over every $J_{k}$, because on $J_{k}$ it differs from $F$ only by a linear function. We thus find $1 / 2^{k}$-decompositions of $\widetilde{F} \mid J_{k}$; more explicitly, there exist $G_{1}^{k}, G_{2}^{k} \in \mathscr{H}\left(J_{k}\right)$ and absolutely continuous $H^{k}: J_{k} \rightarrow \mathbf{R}$, such that the following is satisfied $(k=1,2, \ldots)$ :

(i) $\widetilde{F} \mid J_{k}=G_{1}^{k}-G_{2}^{k}+H^{k}$.

(ii) $G_{2}^{k}$ and $H^{k}$ vanish at the endpoints of $J_{k}$ (and hence, so does $G_{1}^{k}$ by (i) and since $\left.\widetilde{F} \mid P_{0}=0\right)$.

(iii) $\left\|\left(H^{k}\right)^{\prime}\right\|<1 / 2^{k}$.

(iv) $\left\|G_{1}^{k}\right\|_{\infty},\left\|G_{2}^{k}\right\|_{\infty} \leq 2\left\|\widetilde{F} \mid J_{k}\right\|_{\infty}$.

Now define $G_{1}:\left[a_{0}, b_{0}\right] \rightarrow \mathbf{R}$ by

$$
G_{1}:= \begin{cases}G_{1}^{k} & \text { on each } J_{k} \\ 0 & \text { on } P_{0}\end{cases}
$$

and build $G_{2}, H$ analogously. From (iii) we conclude the absolute continuity of $H$ (using Lebesgues dominated convergence theorem). Considering 
(iv) and (ii), a straight-forward argumentation gives the continuity of $G_{1}$ and $G_{2}$. By construction, we have $\widetilde{F}=G_{1}-G_{2}+H$, but this is not yet the desired decomposition. By Lemma 22, we find continuous, n.e. differentiable $W^{k}: J_{k}=\left[a_{k}, b_{k}\right] \rightarrow \mathbf{R}$, fulfilling

(I) $W^{k}\left(a_{k}\right)=W^{k}\left(b_{k}\right)=0$,

(II) $W^{k} \geq \max \left\{\left|G_{1}^{k}\right|,\left|G_{2}^{k}\right|\right\}$,

(III) $\left\|W^{k}\right\|_{\infty}=\left\|\max \left\{\left|G_{1}^{k}\right|,\left|G_{2}^{k}\right|\right\}\right\|_{\infty}\left(\leq 2\left\|\widetilde{F} \mid J_{k}\right\|_{\infty}\right.$ by (iv)).

We construct $W:\left[a_{0}, b_{0}\right] \rightarrow \mathbf{R}$ by

$$
W:= \begin{cases}W^{k} & \text { on each } J_{k} \\ 0 & \text { on } P_{0} .\end{cases}
$$

Again, $W$ is continuous. Let $S_{1}:=G_{1}-W, S_{2}:=G_{2}-W$. By construction, $S_{i}$ is continuous, $S_{i} \leq 0$ and $S_{i} \mid P_{0}=0 \quad(i=1,2)$. We shall show that $S_{1}, S_{2} \in \mathscr{H}\left(\left[a_{0}, b_{0}\right]\right)$ and thus $\widetilde{F}=S_{1}-S_{2}+H$ gives the desired decomposition of $\widetilde{F}$ over $\left[a_{0}, b_{0}\right]$. So the only thing left to show is

$$
D_{-} S_{i}=D^{+} S_{i} \in \mathbf{R} \text { n.e. } \text { on }\left[a_{0}, b_{0}\right](i=1,2) .
$$

Since $S_{i} \mid J_{k}=G_{1}^{k}-W^{k} \in \mathscr{H}\left(J_{k}\right)$ (because $W^{k}$ is n.e. differentiable), we have $D_{-} S_{i}=D^{+} S_{i} \in \mathbf{R}$ n.e. on each $J_{k}$. But $\left\{J_{k}\right\}$ is an at most countable family, so it suffices to show

$$
D_{-} S_{i}=D^{+} S_{i} \in \mathbf{R} \text { n.e. on } P_{0}(i=1,2) \text {. }
$$

But with $J_{k}=\left[a_{k}, b_{k}\right]$, every $x \in P_{0} \backslash\left\{a_{k}, b_{k} \mid k=0,1,2, \ldots\right\}$ is a bilateral accumulation point of $P_{0}$. This, combined with $S_{i} \leq 0$ and $S_{i} \mid P_{0}=0$ forces

$$
D_{-} S_{i}(x)=D^{+} S_{i}(x)=0 \quad(i=1,2)
$$

for our considered $x,\left(^{\prime}\right)$ is shown and the proof is complete.

24. Corollary. The Denjoy integrable functions $f:[a, b] \rightarrow \overline{\mathbf{R}}$ are exactly the members of $\mathscr{L}_{d}^{1}\left([a, b], \mathscr{E}_{d}, I_{d}\right)$ and the Denjoy integral coincides with the $I_{d^{-}}$ integral.

For convenience, let us only sketch the simple proof. Considering Corollary 16 and building indefinite $I_{d}$-integrals, we see that these are exactly functions of the form $F=G+H$ with $G \in \mathscr{G}$ and absolutely continuous $H$. So, by the preceding theorem, these are exactly the functions which are ACG on $[a, b]$, i.e. indefinite Denjoy integrals. Therefore Theorem 18 and Lemma 7 yield that $\mathscr{L}_{d}^{1}$ consists exactly of the Denjoy integrable functions. In either case, the integrals are given as the difference of the indefinite integrals evaluated at the endpoints of the underlying interval, thus the equality of the integrals follows.

At last we shall show that $\mathscr{G}$ and hence $\mathscr{E}_{d}$ are not "too big."

25. Example. There exist absolutely continuous, nondecreasing functions which are not in $\mathscr{G}$.

Proof. By $\lambda$ we denote the Lebesgue measure on the real line. Let $J=[0,1]$ and let $N \subset J$ be an uncountable null set: $\lambda(N)=0$. We find open sets $U_{n}$ with

$$
U_{1} \supset U_{2} \supset U_{3} \supset \cdots, U_{n} \supset N, \quad \lambda\left(U_{n}\right)<\frac{1}{2^{n}} \quad \forall n \in \mathbf{N} .
$$


Setting $h:=\sum_{1}^{\infty} \chi_{U_{n}}$, we have $h \geq 0$ and $h$ is Lebesgue integrable. Thus

$$
H(x):=\int_{0}^{x} h(t) d t \quad(x \in[0,1])
$$

is absolutely continuous and nondecreasing. For arbitrary $x \in N$ and $n_{0} \in \mathbf{N}$ we have

$$
[x, x+\delta] \subset U_{n_{0}} \subset \cdots \subset U_{2} \subset U_{1}
$$

for a suitable $\delta>0$. Hence, for $y \in(x, x+\delta)$ we have

$$
\frac{H(y)-H(x)}{y-x} \geq \frac{1}{y-x} \int_{x}^{y}\left(\sum_{n=1}^{n_{0}} \chi_{U_{n}}(t)\right) d t=n_{0}
$$

and thus $D_{+} H(x) \geq n_{0}$. Since $n_{\jmath} \in \mathbf{N}$ was arbitrary $D_{+} H(x)=\infty$ follows, for $x \in N$. Assuming $H \in \mathscr{G}$, we find $H_{1}, H_{2} \in \mathscr{H}$ with $H=H_{1}-H_{2}$. Since $N$ is uncountable, there must exist $x \in N$ with $D_{-} H_{i}(x)=D^{+} H_{i}(x) \in \mathbf{R}$ $(i=1,2)$. For such an $x$ we can estimate

$$
\begin{aligned}
-D^{+} H_{2}(x) & =D_{+}\left(-H_{2}\right)(x)=D_{+}\left(H-H_{1}\right)(x) \\
& \geq D_{+} H(x)+D_{+}\left(-H_{1}\right)(x) \\
& =D_{+} H(x)-D^{+} H_{1}(x)=\infty,
\end{aligned}
$$

since $D_{+} H(x)=\infty$. But this contradicts $D^{+} H_{2}(x) \in \mathbf{R}$ and the assertion if proved.

\section{BIBLIOGRAPHY}

1. M. Leinert, Daniell-Stone integration without the lattice condition, Arch. Math. 38 (1982), 258.

2. H. König, Integraltheorie ohne Verbandspostulat, Math. Ann. 258 (1982), 447.

3. S. Saks, Theory of the integral, Hafner Publishing Co., New York and Warszawa, 1937.

4. A. Bruckner, Differentiation of real functions, Springer Verlag, Berlin, 1978.

Baeumengass 13, Heidelberg, Germany 